\title{
Path Transmissibility Analysis Considering Two Types of Correlations in Hydropower Stations
}

\author{
Baoping Zhi and Zhenyue Ma \\ School of Hydraulic Engineering, Faculty of Infrastructure Engineering, Dalian University of Technology, Room 330, \\ Building 3 of Lab, Dalian, Liaoning Province 116023, China
}

Correspondence should be addressed to Zhenyue Ma; dmzy@dlut.edu.cn

Received 16 May 2013; Accepted 4 August 2013

Academic Editor: Daniel Dias-da-Costa

Copyright (C) 2013 B. Zhi and Z. Ma. This is an open access article distributed under the Creative Commons Attribution License, which permits unrestricted use, distribution, and reproduction in any medium, provided the original work is properly cited.

A new vibration model is built by introducing the head-cover vibration transfer path based on a previous analysis of the vertical vibration model for hydropower station units and powerhouses. This research focuses on disturbance- and parameter-related transfer paths in a practical situation. In a complex situation, the application of the stochastic perturbation method is expanded using an algebra synthesis method the Hadamard product, and theoretical analyses, and numerical simulations of transfer paths in the new vibration model are carried out through the expanded perturbation method. The path transfer force, the path transmissibility, and the path disturbance ranges in the frequency domain are provided. The results indicate that the methods proposed in this study can efficiently reduce the disturbance range and can accurately analyze the transfer paths of hydraulic-source vertical vibration in hydropower stations.

\section{Introduction}

Hydraulic vibration is the main vibration source in hydropower station units and powerhouses. The vertical vibration in units is usually caused by hydraulic pressure fluctuations or other loads on the water turbine's flow passage components. Field and model tests have shown that there are three main vibration transfer paths running from the water turbine to the powerhouse [1]: (1) runner-shaft-bearing-fixed components (machine frame, head-cover-powerhouse;) (2) flow pressurespiral case-powerhouse; and (3) runner-runner negative pressure region-head cover-powerhouse. Previous studies concerning the vertical vibration produced by a hydraulic source mainly focus on path (1), while the effects of paths (2) and (3) are usually ignored [2]. However, as the scale and capacity of hydropower stations increase, the flow passage area of the head-cover system continually increases, with corresponding increases in the vibration of the head-cover system. Therefore, the influence of the head-cover system becomes more important in the hydraulic vibration transfer path, and ignoring path (3) will produce a larger error. Therefore, it is imperative that the contribution of path (3) should be analyzed; more specifically, the contribution of the vibration of the head-cover system of the hydropower station vertical vibration transfer should be analyzed. However, due to the presence of randomness, it is difficult to clearly and accurately describe the contribution of this transfer path to the structural vibration.

Theoretical analyses of the vibration transfer path can be classified as a stochastic structural system problem. At present, the Monte-Carlo numerical simulation method (MCSM) $[3,4]$ and the perturbation method [5-7] are the most popular analysis methods. MCSM is used less frequently because of the large amount of computation required when dealing with a large-scale structure. Conversely, the perturbation method is applied by many researchers in various fields. Collins and Thompson [8] initially employed the perturbation method to analyze stochastic dynamical systematic characteristics in 1969; the perturbation method was later employed in a static analysis by Hisada and Nakagiri [9] and in a dynamic analysis by Liu et al. [10]. Kronecker algebra was introduced to the expansion of the perturbation method by Vetter [11]. After several decades of development, relevant studies on perturbation theory were quite abundant. The primary methods included the L-P method [12], the multiple scale method $[13,14]$, the average method [15], the KBM 
(Krylov-Bogoliubov-Mitropolsky) method [15, 16] and the singular perturbation method $[17,18]$. Recent developments include the homotopy perturbation method [19]. In the analysis of transfer paths combined with the perturbation method, Zhang et al. built a theoretical model for vibration transfer path analysis by implementing the perturbation method with Kronecker algebra in the static analysis [20], the dynamic analysis [21] and the reliability analysis [22, 23]. This model has been used for the analysis and design of mechanical components. Computations of the transfer path considering multi-vibration sources have been carried out by Zhao and Zhang [24] based on Zhang's research. These results have been used for isolation vibration analysis. The above research studies focus on additive disturbance analysis. Using multiplicative disturbance analysis, Gao et al. separately analyzed the dynamical characteristics of a truss structure by using the interval factor method [25], the random factor method [26], and nonstationary random excitation [27]. Ma et al. conducted a dynamical characteristic analysis of a linear [28] and nonlinear [29] truss structure using fuzzy variables. In 2010, a two-factor method was proposed by Ma et al. [30], which considers two multiplicative disturbances. However, because of the limitations of these methods, they have not been applied to transfer path analysis with respect to a multiplicative disturbance. Furthermore, previous studies have not jointly analyzed these two types of disturbances. In practice, the two types of disturbances always exist together, and there are some correlations between these two disturbances. There are some correlations among the parameters of the structure as well. Thus, the previous studies were not based on an accurate analysis because they only considered a single disturbance, neglected the correlations between disturbances, or neglected the correlations among parameters. Therefore, it is necessary to jointly analyze the problem of the vibration transfer path of time-invariable parameters for the two types of disturbances and their related parameters. Only a few studies have reported on the two types of correlations in perturbation theory. Previous studies on correlations ignored the two types of correlation or converted relevant variables into irrelevant variables. These analyses did not consider the effects of correlations and thus were not rigorous. At present, only a few researchers have attempted to explore this aspect. Some examples include studies on molecular chemistry [31], nuclear physics [32], and vocal vibration [33, 34]. Of these studies, only the study on vocal vibration considered the correlation parameters, but it did not involve the perturbation method. At present, there are few available studies on structural analysis. Pirrotta [35] conducted a perturbation analysis of delta-correlated processes, but he did not study the correlation between parameters; Ambrogio [36] described the correlations by considering the correlation coefficient between additive and multiplicative disturbances for one parameter, but he also did not refer to the correlation between parameters. Husain et al. [37] solved the problem of parameter correlation, but they only considered computational results and did not extend their results to improve the perturbation method. Khodaparast et al. [38] corrected the structure variation model, but they ignored the correlation between modified parameters and measurement values. This study focuses on the application of perturbation theory with two types of correlation from the perspective of structure analysis.

Hydropower station units and powerhouses are largescale structures. Most of the parameters' disturbances should be less than $10 \%$ of their mean values in such a large structure. Therefore, problems regarding hydropower stations can be solved by the perturbation method. First, the head-cover system is introduced, which has the same vibration source as a path (1); the elastic foundation constraint is selected; and a new vibration model is built on the basis of the previous vertical vibration model. Furthermore, a method for solving the path transfer force is proposed using the general method of dynamic analysis. Second, for the test signal, correlations between the two types of disturbances and correlations between the parameters are considered based on the single disturbance vibration path analysis. By using the coefficient of variation algebra synthesis method [39], Kronecker algebra [11], and the Hadamard product [40], gradient-sorting estimations of the transfer paths in the frequency domain are carried out, and methods for determining the transfer force and transmissibility and their disturbances are proposed. Finally, the method described in this paper is verified using the model of a large hydropower station.

\section{Analysis Model with the Introduction of a Head-Cover System}

For a hydroelectric generating unit, regardless of whether it has a suspension or umbrella structure, the weight of its rotating parts is successively transferred to the reinforced concrete machine foundation through the thrust bearing, frame (suspension units containing the stator frame), and sole screw. The head-cover system is always fixed on the base ring strengthening plate, and the head-cover system and the strengthening plate are considered to be one part. Taking a vertical vibration characteristic analysis of the umbrella unit as an example, the model contains a shaft system, thrust bearing, and lower bracket (see Figure 1). The heave shaft can be simplified as a massless elastic continuous beam, and then its mass can be regarded as a mass attached to three nodes, $m_{1}$, $m_{2}$, and $m_{3} . m_{1}$ can be defined as the mass of the excitation rotor and the shaft, the half shafting mass, which is measured from the heavy shaft top to the rotor frame, and another mass added on top of the heavy shaft; $m_{2}$ can be defined as the mass of the central body of the rotor frame, the half mass of the whole gate arm, and the half mass of the whole shaft; and $m_{3}$ can be defined as the mass of the water, turbine runner, the additional mass of water and the half shafting mass, which is measured from the rotor frame to the hydraulic turbine. The rotor gate arm can be simplified as a massless elastic continuous rod, and then its mass can be assigned to the runner margin and the central body of the rotor frame. $k_{4}$ can be defined as the sum of the vertical stiffness of the whole gate arm, and $m_{4}$ can be defined as the lumped mass of the runner margin. The outer end of the lower bracket is fixed to the concrete foundation. The lower bracket gate arm can then be simplified as a gravity-free beam if the coupling effect of the foundation is ignored. $k_{52}$ can be defined as the vertical 


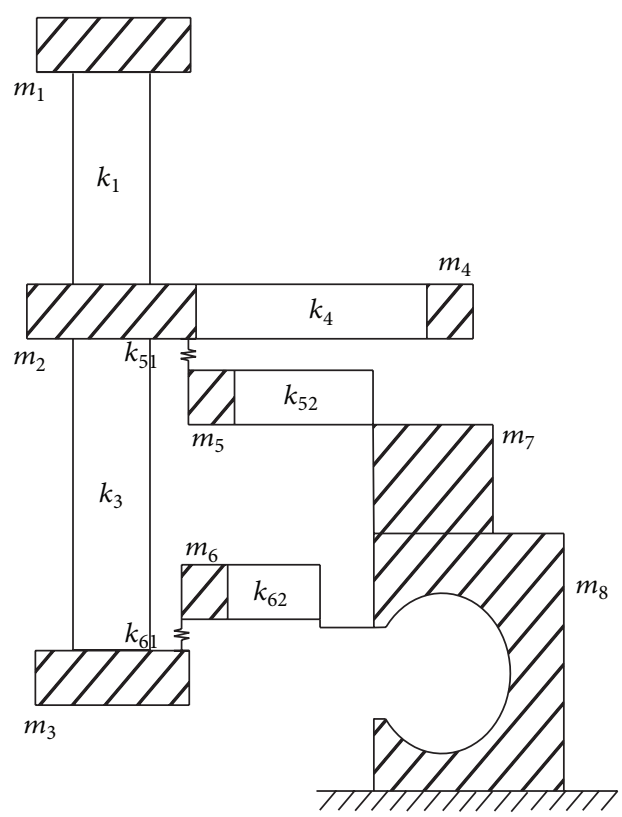

FIGURE 1: Simplified model of the coupling system between the umbrella unit and the powerhouse.

stiffness of the lower bracket gate arm. $m_{5}$ can be defined as the lumped mass of one end of the lower bracket, which is close to the heavy shaft, and the half mass of the gate arm; $m_{5}$ can be connected to $m_{2}$ by the thrust bearing, which is simplified by an equivalent stiffness $k_{51}$.

With the introduction of the head-cover system, the vibration transfer path is as follows: first, the hydraulic vertical vibration is transferred to the head cover by the heavy shaft seal and guide bearing in the water turbine runner chamber; next, the vibration is transferred from the head cover to the spiral case base ring strengthening plate, which is connected to the outer end of the head cover; and finally, the vibration is transferred to the machine foundation by the wrapped concrete outside the spiral case. Ignoring the coupling effect, the control parts and other additional parts on the head cover can be regarded as the attached mass of the head-cover system. As the lumped mass is close to the heavy shaft, $m_{6}$ can be defined as the mass of the central body and the half mass of the whole head-cover system. The head-cover system can be simplified as a gravity-free beam, and $k_{62}$ can be defined as the vertical stiffness. $m_{6}$ can be connected to $m_{3}$ by the sealing spring, which lies between the head-cover structure and the water turbine runner. The vertical stiffness of the connection can be simplified by an equivalent stiffness $k_{61}$.

$k_{51}$ is the series stiffness of the elastic oil tank stiffness (thrust bearing support system) and the oil film stiffness. $k_{61}$ is the series stiffness of the sealing structure stiffness and the clearance water stiffness. These two parameters exhibit a linear relationship because the unit's axial water thrust varies with the unit's conditions. However, $k_{51}$ and $k_{61}$ are simplified as a single stochastic variable in this study.

In the process of examining the unit's vertical vibration, the machine foundation pier can be regarded as an elastic foundation. The structure can be treated as a single node, $m_{7}$, which ranges from the machine foundation pier to the concrete floor in the turbine layer. The structure can be treated as a single node, $m_{8}$, which ranges from the turbine layer to the foundation. The stiffness of this structure is replaced by the equivalent stiffness, which is based on the strengthening plate, spiral structure, and concrete structure. Thus, the hydropower station base is treated by dividing it into two nodes at the connection of the head-cover system and the hydropower station base, where $k_{62}$ is only connected to $m_{8}$.

For the entire model, the form and meaning of the dampness matrix are similar to those of the stiffness matrix. Assuming that the system is linear, the differential equation of the vibration is found from the Lagrange equation:

$$
\mathbf{M u ̈}+\mathbf{C} \dot{\mathbf{u}}+\mathbf{K u}=\mathbf{F}(t) .
$$

By merging these dynamical balance equations for the shaft, the rotor, the lower bracket, the head-cover system, and the machine foundation pier, an equation with 8 degrees of freedom and 26 parameters can be obtained, and the total stiffness matrix can be written as

$$
\mathbf{K}=\left(\begin{array}{cccccccc}
k_{1} & -k_{1} & 0 & 0 & 0 & 0 & 0 & 0 \\
-k_{1} & k_{1}+k_{3}+k_{4}+k_{51} & -k_{3} & -k_{4} & -k_{51} & 0 & 0 & 0 \\
0 & -k_{3} & k_{3}+k_{61} & 0 & 0 & -k_{61} & 0 & 0 \\
0 & -k_{4} & 0 & k_{4} & 0 & 0 & 0 & 0 \\
0 & -k_{51} & 0 & 0 & k_{51}+k_{52} & 0 & -k_{52} & 0 \\
0 & 0 & -k_{61} & 0 & 0 & k_{61}+k_{62} & 0 & -k_{62} \\
0 & 0 & 0 & 0 & -k_{52} & 0 & k_{52}+k_{7} & -k_{7} \\
0 & 0 & 0 & 0 & 0 & -k_{62} & -k_{7} & k_{62}+k_{7}+k_{8}
\end{array}\right) .
$$

The total mass matrix is obtained from the lumped mass:

$$
\mathbf{M}=\operatorname{diag}\left\{m_{1}, m_{2}, m_{3}, m_{4}, m_{5}, m_{6}, m_{7}, m_{8}\right\} .
$$

Generally, the characteristics of the vertical vibration source in the hydropower station are unique position, simple contact surface with structure, and few ingredients. Conversely, the characteristics of the lateral (radial) vibration are multipositions, strong nonlinear contact surface, and complex excitation. For simplifying the model and the calculation, the total structure of the unit's powerhouse is considered as suffering vertical harmonic excitation. The vertical 
harmonic excitation is located on the water turbine runner, and the response of the total structure is in a steady state. In addition, the initial phase remains constant throughout the whole process. Setting the steady-state response as $u_{i}(t)=$ $\bar{U}_{i} e^{i\left(\omega t+\varphi_{i}\right)}$ and $U_{i}=\bar{U}_{i} e^{i \varphi_{i}}$ yields

$$
\begin{gathered}
u_{i}(t)=U_{i} e^{i \omega t}, \quad \dot{u}_{i}(t)=i \omega U_{i} e^{i \omega t}, \\
\ddot{u}_{i}(t)=-\omega^{2} U_{i} e^{i \omega t}, \\
\mathbf{U}=\left\{u_{1}, u_{2}, u_{3}, u_{4}, u_{5}, u_{6}, u_{7}, u_{8}\right\}^{T}, \\
\mathbf{F}(t)=\left\{0,0, F_{0} e^{i \omega t}, 0,0,0,0,0\right\}^{T} .
\end{gathered}
$$

Substituting (4a)-(4c) into (1) gives the dynamical balance equation of the hydropower station's vertical vibration with the head-cover system in the frequency domain:

$$
\left(-\omega^{2} \mathbf{M}+i \omega \mathbf{C}+\mathbf{K}\right) \mathbf{U}=\mathbf{F}(t) \text {. }
$$

The response vector $\mathbf{U}$ at each node of the structure was obtained by numerical calculations. $F_{\text {zhou }}$ and $F_{\text {ding }}$ denote the forces that are transferred to the machine foundation pier from the water turbine by the shaft system and the head-cover system, respectively, and can be written as

$$
\begin{aligned}
F_{\text {zhou }} & =k_{\text {zhou }}\left(u_{3}-u_{7}\right)+c_{\text {zhou }}\left(\dot{u}_{3}-\dot{u}_{7}\right) \\
& =\left(k_{\text {zhou }}+i \omega c_{\text {zhou }}\right)\left(U_{3}-U_{7}\right) e^{i \omega t}, \\
F_{\text {ding }} & =k_{\text {ding }}\left(u_{3}-u_{7}\right)+c_{\text {ding }}\left(\dot{u}_{3}-\dot{u}_{7}\right) \\
& =\left(k_{\text {ding }}+i \omega c_{\text {ding }}\right)\left(U_{3}-U_{7}\right) e^{i \omega t} .
\end{aligned}
$$

In (6a)-(6b), $k_{\text {zhou }}$ and $c_{\text {zhou }}$ denote the path stiffness and the path dampness of the vibration path through the shaft system, respectively. Similarly, $k_{\text {ding }}$ and $c_{\text {ding }}$ denote the path stiffness and the path dampness through the head-cover system, respectively. When calculating the path stiffness and dampness of the shaft system, $m_{1}$ and $m_{4}$ are treated as dynamic vibration absorbers with dampness.

\section{Vibration Path Sorting Considering Two Types of Correlations}

In the analysis of a hydropower station, parameters such as the stiffness, mass, and dampness are attributed to the multiplicative disturbances arising from the material properties, manufacturing technology, and other factors. These parameters, obtained by measurement, are attributed to the additive disturbances due to the testing noise and environmental noise. Furthermore, because the testing method and environment are the same for every parameter, the two disturbances of each parameter are interrelated. These parameters are also interrelated by means of continuous structure discretization.

Given the factors mentioned above, the mass, stiffness, and dampness are described as a random vector a with $n$ random variables, where every random variable involves two types of disturbances. When the variable disturbance is lower than $15 \%$ of the mean value, the stochastic variable can be expressed as

$$
a_{i}=a_{i}^{1} a_{i}^{d}+a_{i}^{2}
$$

In (7), $a_{i}$ denotes the $i$ th element in the random vector $\mathbf{a}$. $a_{i}^{1}$ and $a_{i}^{2}$ denote the multiplicative and additive disturbance of the random variable $a_{i}$, respectively. Assuming that their mean values are 1 and 0 , respectively, $a_{i}^{d}$ denotes the deterministic component of $a_{i}$, which represents the mean value after multiple samplings.

According to the coefficient of variation algebra synthesis method [40], calculating the mathematical expectation and variance of (7) yields

$$
\begin{gathered}
E_{a_{i}}=E\left[a_{i}^{1} a_{i}^{d}+a_{i}^{2}\right]=E\left(a_{i}^{1} a_{i}^{d}\right)+E\left(a_{i}^{2}\right)=a_{i}^{d}, \\
\sigma_{a_{i}}^{2}=\operatorname{Var}\left(a_{i}\right)=E\left[\left(a_{i}-E_{a_{i}}\right)^{2}\right] \\
=E\left[\left(\left(a_{i}^{1}-a_{i}\right) a_{i}^{d}+a_{i}^{2}\right)^{2}\right] \\
=\left(a_{i}^{d}\right)^{2} \sigma_{a_{i}^{1}}^{2}+\sigma_{a_{i}^{2}}^{2}+2 a_{i}^{d} \operatorname{Cov}\left(a_{i}^{1}, a_{i}^{2}\right),
\end{gathered}
$$

where

$$
\operatorname{Cov}\left(a_{i}^{1}, a_{i}^{2}\right)=\rho_{a_{i}^{1}, a_{i}^{2}} \sigma_{a_{i}^{1}} \sigma_{a_{i}^{2}},
$$

where $\rho_{a^{1}, a^{2}}$ is the correlation coefficient of the multiplicative and additive disturbances contained in the parameter $a$. If these parameters are interrelated, then

$$
\operatorname{Cov}\left(a_{i}, a_{j}\right)=\rho_{a_{i}, a_{j}} \sigma_{a_{i}} \sigma_{a_{j}}
$$

where $\rho_{a_{i}, a_{j}}$ is the correlation coefficient of the random variables $a_{i}$ and $a_{j}$. Generally, if the random variables follow a normal distribution, their linear transformations and multiplication will also follow a normal distribution. For example, if the vector a follows a normal distribution, then the function $F_{i}($ a) will follow a normal distribution. Using the Taylor expansion, expanding the transfer force $F_{i}$ at the mean value $F_{i}^{d}$ yields

$$
\begin{aligned}
F_{i} & =F_{i}^{d}+\frac{\partial F_{i}^{d}}{\partial \mathbf{a}^{T}}\left(\mathbf{a}-\mathbf{a}^{d}\right)+O\left(\mathbf{a}^{p}\right) \\
& =F_{i}^{d}+\frac{\partial F_{i}^{d}}{\partial \mathbf{a}^{T}} \mathbf{a}^{p}+O\left(\mathbf{a}^{p}\right),
\end{aligned}
$$

where

$$
\mathbf{a}^{p}=\mathbf{a}-\mathbf{a}^{d}=\left(\mathbf{a}^{1}-1\right) \mathbf{a}^{d}+\mathbf{a}^{2} .
$$

Neglecting components of the second order and above, the disturbance of the transfer force is

$$
F_{i}^{p}=\sum_{k=1}^{m} \frac{\partial F_{i}^{d}}{\partial a_{k}} a_{k}^{p} .
$$


In (13), $\partial F_{i}^{d} / \partial a_{k}$ is the partial derivative of $F_{i}$ with respect to the random variable $a_{k}$, which is also the first-order sensitivity of $a_{k}$. The covariance of $F_{i}^{p}$ and $F_{j}^{p}$ is

$$
\begin{aligned}
\operatorname{Cov}\left(F_{i}, F_{j}\right) & =E\left[F_{i}^{p} F_{j}^{p}\right] \\
& =E\left[\left(\sum_{k=1}^{m} \frac{\partial F_{i}^{d}}{\partial a_{k}} a_{k}^{p}\right)\left(\sum_{l=1}^{m} \frac{\partial F_{j}^{d}}{\partial a_{l}} a_{l}^{p}\right)\right] .
\end{aligned}
$$

That is,

$$
\begin{aligned}
\operatorname{Cov}\left(F_{i}, F_{j}\right) & =\sum_{k=1}^{m} \sum_{l=1}^{m} \frac{\partial F_{i}^{d}}{\partial a_{k}} \frac{\partial F_{j}^{d}}{\partial a_{l}} E\left(a_{k}^{p} a_{l}^{p}\right) \\
& =\sum_{k=1}^{m} \sum_{l=1}^{m} \frac{\partial F_{i}^{d}}{\partial a_{k}} \frac{\partial F_{j}^{d}}{\partial a_{l}} \operatorname{Cov}\left(a_{k}, a_{l}\right) .
\end{aligned}
$$

Equation (16) shows that $\operatorname{Cov}\left(F_{i}, F_{j}\right)$ of the transfer force $F_{i}$ and $F_{j}$ can be expressed by $\operatorname{Cov}\left(a_{k}, a_{l}\right)$ of the random structure parameter:

$$
\operatorname{Cov}\left(F_{i}, F_{j}\right)=\sum_{i=1}^{m} \sum_{j=1}^{m} \frac{\partial F_{i}^{d}}{\partial a_{k}} \frac{\partial F_{j}^{d}}{\partial a_{l}} \rho_{a_{k}, a_{l}} \sigma_{a_{k}} \sigma_{a_{l}} .
$$

$$
\begin{aligned}
& \text { If } i=j, \\
& \qquad \sigma_{F_{i}}^{2}=\operatorname{Cov}\left(F_{i}, F_{i}\right)=\sum_{k=1}^{m} \sum_{l=1}^{m} \frac{\partial F_{i}^{d}}{\partial a_{k}} \frac{\partial F_{i}^{d}}{\partial a_{l}} \rho_{a_{k}, a_{l}} \sigma_{a_{k}} \sigma_{a_{l}} .
\end{aligned}
$$

According to Kronecker algebra [11], the corresponding stochastic analysis theory and Hadamard product [41] yield

$$
\sigma_{F_{i}}^{2}=\left|\frac{\partial F_{i}^{d}}{\partial \mathbf{a}^{T}}\right|^{[2]}\left(\overrightarrow{\boldsymbol{\rho}} \circ\left(\sigma_{\mathbf{a}}\right)^{[2]}\right),
$$

where the subscript [2] denotes the Kronecker power; that is, $a^{[2]}=a \otimes a$; if $a$ is of order $n \times 1$, then $a^{[2]}$ is of order $n^{2} \times 1 . \vec{\rho}$ is the stacking vector of the matrix of correlation coefficients, which is also of order $n^{2} \times 1$. The symbol $\circ$ denotes the Hadamard product. Therefore, $\sigma_{F_{i}}^{2}$ is of order $1 \times 1$.

The sensitivity matrix $\left|\partial F_{i}^{d}(a) / \partial \mathbf{a}^{T}\right|$ of the transfer force of each parameter is

$$
\left|\frac{\partial F_{i}^{d}}{\partial \mathbf{a}^{T}}\right|=\left|\frac{\partial F_{i}^{d}}{\partial a_{1}} \cdots \frac{\partial F_{i}^{d}}{\partial a_{n}}\right| .
$$

Substituting (19) into (18), the variance of the transfer force $F_{i}$ for each path can be calculated. Equation (11) only involves the first-order Taylor expansion. A higher-order Taylor expansion will improve the accuracy, but it involves complicated mathematical calculations. Equation (18) shows that the transfer force variance can be directly obtained from the random variables' numerical characteristics. The calculations can thus be simplified because the sample is not included in the mathematical operations. The correlations between parameters are merely modified in (18) and do not increase the number of calculations required. The transmissibility is defined as the ratio of the amplitudes between the transfer force and the vibration source excitation force:

$$
\beta_{i}=\left|\frac{F_{i}}{F_{0}}\right| \text {. }
$$

Neglecting the disturbance of the excitation force $F_{0}$, based on the random variable algebra synthesis method, the expectation, variance, and transfer coefficient of the transmissibility can be written as

$$
\begin{gathered}
E_{\beta}=E[\beta(a)]=\frac{E\left(F_{t}\right)}{E\left(F_{0}\right)}, \\
\sigma_{\beta}^{2}=\frac{E\left[F_{i}\right]^{2}}{E\left[F_{0}\right]^{2}}\left|\frac{\operatorname{Var}\left[F_{i}\right]}{\left[E\left(F_{i}\right)\right]^{2}}\right|, \\
\theta=\frac{E_{\beta}}{\sigma_{\beta}} .
\end{gathered}
$$

The result of a random variable that follows a normal distribution divided by a constant also follows a normal distribution. $E\left[F_{0}\right]$ and $E\left[F_{i}\right]$ are known in the deterministic system, so the variance of the transmissibility will follow a normal distribution. The transfer coefficient is the transfer efficiency of the excitation force. The gradient sorting of the vibration path transmissibility in the frequency domain can be obtained by changing the frequency of the excitation force.

A solution of the vibration transfer path for the two types of disturbances and correlations is proposed based on the above methods. The path transmissibility and its probabilistic characteristic are provided. In this study, the solution only involves the first-order sensitivity of the random parameter and the probabilistic characteristic of the random variable. Additionally, the introduction of the two types of correlations does not involve excessive calculations. Therefore, the calculation accuracy is improved for practical problems.

\section{Example Analysis}

The main structure of the umbrella unit in a large hydropower station is shown in Figure 2, and the simplified model of transfer path is shown in Figure 1. In this example, the effect of the spiral case and substructure is ignored $[41,42]$ because their effect is far less than that of the upper structure. The excitation is assumed to be a simple harmonic excitation. The mean values of the random parameters can be obtained from the hydropower station design diagrams. These mean values are $m_{1}=8.28 \times 10^{4}, m_{2}=1.042 \times 10^{6}, m_{3}=3.29 \times 10^{5}$, $m_{4}=9 \times 10^{5}, m_{5}=1.2 \times 10^{5}, m_{6}=1.15 \times 10^{5}, m_{7}=$ $1.39 \times 10^{5}$ and $m_{8}=8.92 \times 10^{5}$, in units of kg; $k_{1}=7.26 \times 10^{10}$, $k_{3}=5.72 \times 10^{10}, k_{4}=2.32 \times 10^{10}, k_{51}=2.20 \times 10^{12}$, $k_{52}=9.41 \times 10^{9}, k_{61}=1.73 \times 10^{8}, k_{62}=1.73 \times 10^{10}$, $k_{7}=7.70 \times 10^{9}$, and $k_{8}=4.26 \times 10^{8}$, where the unit of the stiffness $k$ is N/m; and $c_{1}=5.48 \times 10^{6}, c_{3}=4.11 \times 10^{6}, c_{4}=$ $1.02 \times 10^{7}, c_{51}=2.57 \times 10^{7}, c_{52}=7.51 \times 10^{5}, c_{61}=2.23 \times 10^{5}$, $c_{62}=9.99 \times 10^{4}, c_{7}=1.64 \times 10^{6}$, and $c_{8}=9.74 \times 10^{5}$, where the unit of the dampness $c$ is $\mathrm{N} \cdot \mathrm{s} / \mathrm{m}$. Each parameter comprises 


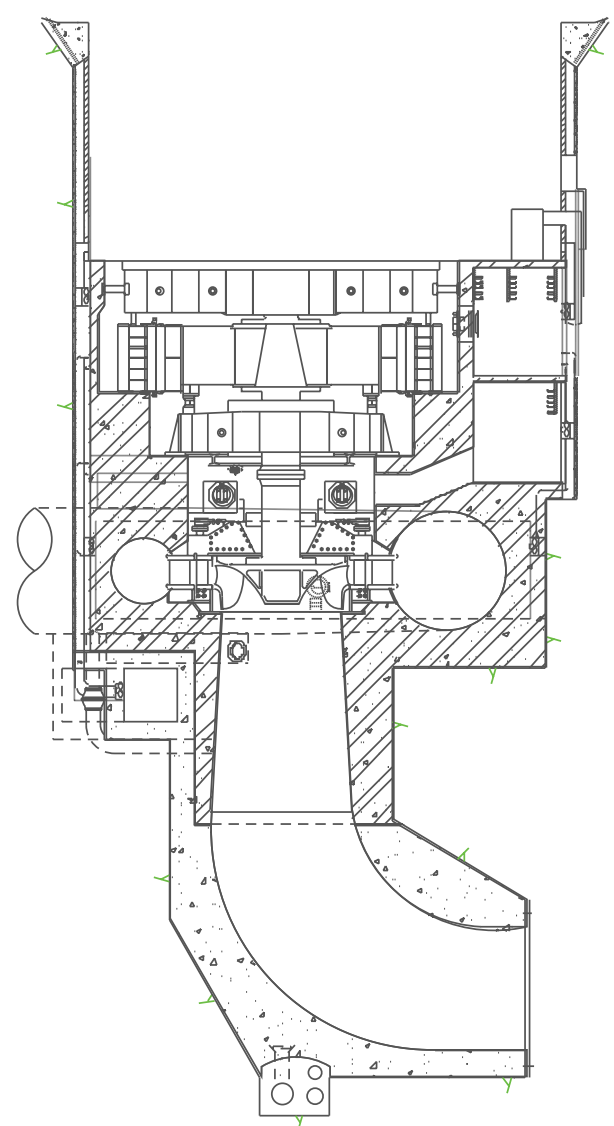

Figure 2: Cutaway view of the main structure of hydropower.

two disturbances that follow a normal distribution. The multiplicative disturbance is related to the difficulty of obtaining the parameters. The multiplicative variance coefficients of $k_{51}$ (including the vertical stiffness of the thrust bearing) and $k_{61}$ (the sealing equivalent vertical stiffness between the head cover and runner) are set to 0.10 . The equivalent bending rigidity of $k_{62} \mathrm{~s}$ multiplicative variance coefficient is set to 0.075 because there are many uncertain factors in the control components and other attached components on the head cover. The multiplicative random variance coefficients of the other parameters are set at 0.05 . The additive disturbance is related to the measurement range. The standard deviations of the additive random variable with respect to mass and stiffness are set at $10^{4}$ and $10^{8}$, respectively, according to each parameter's mean value. The variance coefficient of damping is similar to that of the mass and stiffness. In this example, the parameters' disturbances are generated by a function in the MATLAB software, and the sample size is 10000 for each disturbance. All errors are determined to be smaller than $0.1 \%$ by comparing the variances and mean values of the generated samples with the corresponding set values. Therefore, the generated sample variances and mean values are used in the study. The correlation function is determined by the generated function. The partial derivative is calculated by the software Mathematica. The coefficient correlation of the generated sample is 0.0329 .

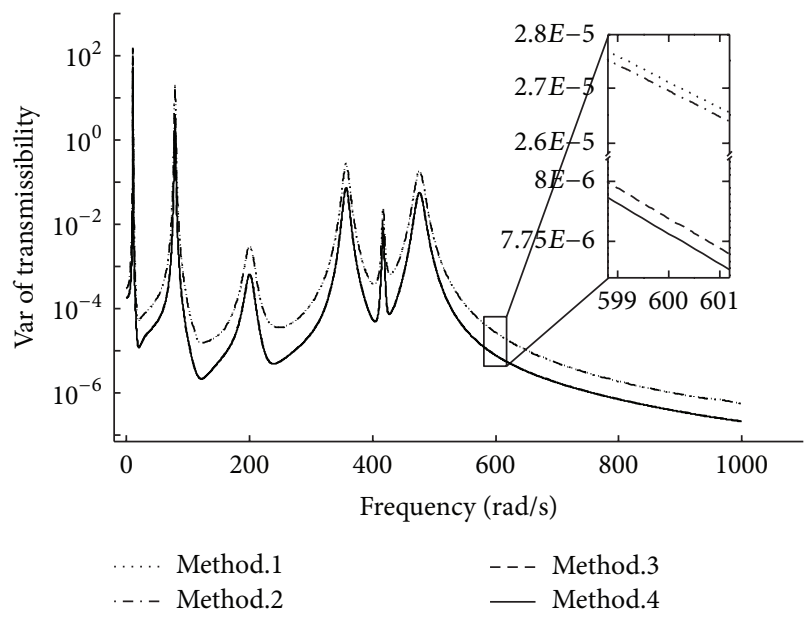

FIGURE 3: Head-cover transmissibility variances in the frequency domain as predicted by the four methods.

Figure 3 displays the transmissibility variances of the head-cover transfer path obtained using different methods. Method 1 does not involve the two types of correlations. The Monte-Carlo simulation is not used in method 1 (due to the inclusion of 26 random variables and because the calculation time is too long, this method only focuses on how each group of variables changes with time, that is, the number of calculations is equal to the sample size). Method 2 (following the method developed by W. J. Vetter in 1973) only involves the correlation with respect to one parameter, which involves the correlations between the disturbances of the parameters. Method 3 (similar to method 2) involves the correlations between parameters instead of the correlations between the disturbances of the parameters. Method 4 involves both types of correlations. The results for method 4 are obtained from (18). Figure 3 displays the characteristic curve of the headcover system transmissibility variances in the frequency band of $0-1000 \mathrm{rad} / \mathrm{s}$. Figure 4 presents the optimal curve of the two types of correlations for the transmissibility variance range as the correlation coefficient changes. This figure contains two curves: the disturbance curve, which describes the mean values of the results of method 2 subtracted from method 1 and the results of method 4 subtracted from method 3, and the parameter curve, which is similar to the disturbance curve.

Figures 3 and 4 show the following results: (1) compared to the other methods, the variance range for the method that contains two types of correlations is the smallest. This finding indicates that this method significantly decreases the disturbance range under theoretical calculations. The path transmissibility variance decreases by $72.51 \%$. (2) In optimizing the method considering correlations between the disturbances and variances, the range merely decreases by $0.644 \%$. This small decrease may have arisen because the difference between the additive and multiplicative disturbances is large, and the optimizing function is weak. (3) The optimization effect is significant for the method considering the correlations between parameters. The variance range 


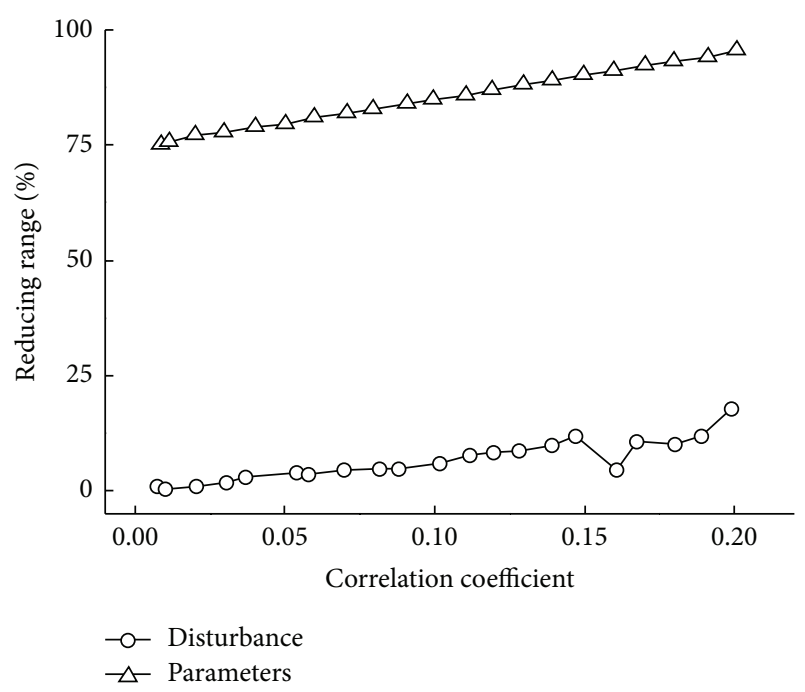

Figure 4: Optimization range of the correlation coefficient characteristic curve.

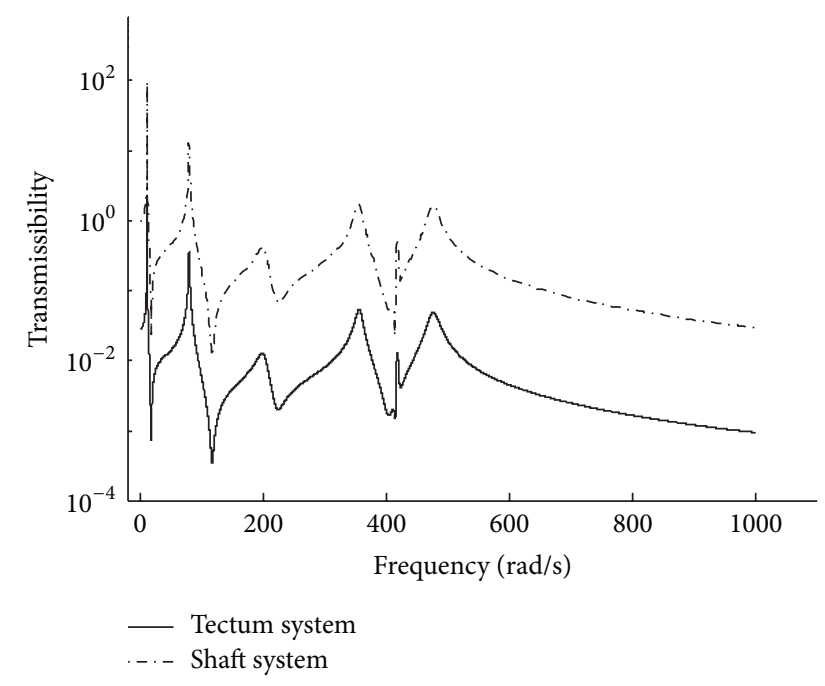

FIgURE 5: Path transmissibility of the excitation frequency curve.

decreases by $75.71 \%$ when the correlation coefficient is 0.0085 . Thus, neglecting this type of correlation leads to an inaccurate analysis. (4) As the correlation coefficient increases, the optimization of the transmissibility variance is more efficient, and the effect on the disturbance range is more significant. This result demonstrates that the relationship between the optimization range and the correlation coefficients for the parameters is linear and that the relationship between the optimization range and the correlation coefficients for the disturbances is proportional. However, the latter relationship does exhibit some fluctuations.

Figure 5 compares the characteristic curve for each path transmissibility, $\beta_{i}$, to the excitation frequency. This curve shows the ratio between the forces that are transmitted to the machine pier by the corresponding paths and the excitation force of the water turbine hydraulic source. This ratio is

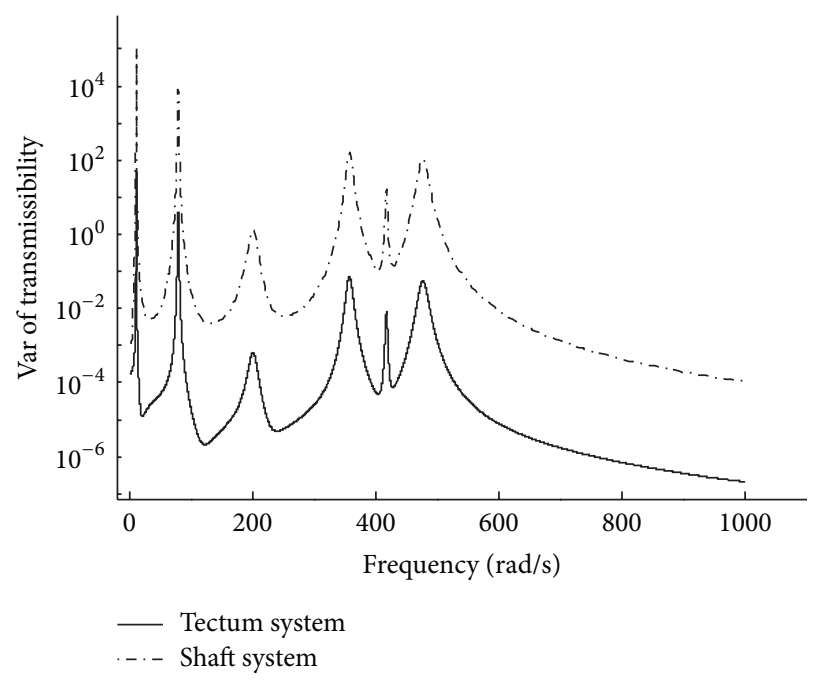

FIGURE 6: Variance of the path transmissibility of the excitation frequency characteristic curve.

equivalent to the amplification coefficient. The curve indicates two results: (1) resonance occurs when the frequency of the excitation force is the same as the natural frequency. The transmissibility reaches its maximum value for each path at this frequency. This result indicates that the path has a large effect on the structural vibration at this frequency. (2) Considering the whole process of vertical vibration transfer, the transmissibility of the head cover is significantly smaller than that of the shafting system. Throughout the entire frequency range, the mean value of the path transmissibility ratio is 32.37. This value indicates that the vibration effect of the headcover system can be neglected in accurate calculations.

Figure 6 shows that the characteristic curve of the transmissibility variance varies with the excitation frequency for the two types of correlations. This curve reflects the diversion of the transmissibility and indicates the following findings. (1) The path transmissibility variance of the shaft system is greater than that of the head-cover system due to the large number of components and the randomness of these components' parameters. Thus, the variance of a structure with a high level of parameter randomness is large in the transfer path. (2) The variance maximum occurs at the natural frequency. This frequency is determined by the structural parameter, which accounts for the parameter contribution of every transfer path, enabling the transfer force to be obtained from the expectation and variance of the transmissibility.

Figure 7 shows that the characteristic curve of the transfer coefficients varies with the excitation frequency for the two types of correlations. This curve reflects the transfer efficiency of the excitation force. The figure shows that the transfer efficiency of the head-cover system is more efficient than that of the shafting system. Therefore, more attention should be given to the assigned vibration proportion of the shaft and head-cover system in a design. 


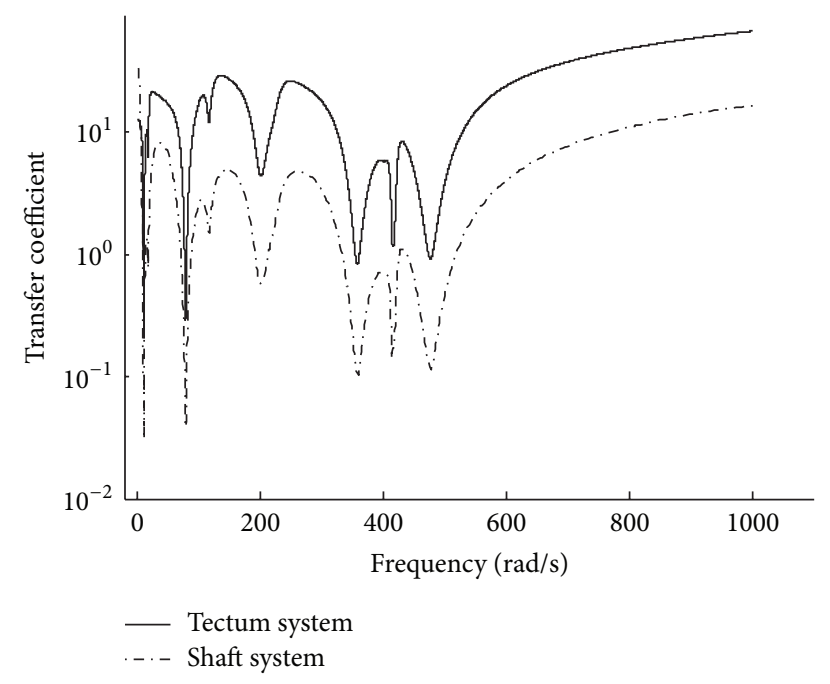

Figure 7: Transfer coefficient of the paths of the excitation frequency characteristic curve.

\section{Conclusions}

(1) The multivibration source, multipath vibration model, is improved with respect to the coupling effect between hydropower station units and powerhouses by the introduction of a head-cover system. The scalar expression of each vibration transfer path is provided in this study.

(2) A perturbation analysis of the transfer path is carried out by using a test signal. A complex situation with two types of correlations is considered. Kronecker algebra, the Hadamard product, and probabilistic statistics are employed to develop an analysis method for the transfer path. In the solution process, excessive additional computations are not required because only the numerical characteristics of the random variables are used. The application of the stochastic perturbation method is expanded, and a method for analyzing the vibration transfer path is developed. The analysis method efficiently decreases the disturbance range of the path's contribution. The disturbance ranges of the path transmissibility and the contribution rate are efficiently reduced by considering the two types of correlations. This reduction is significant for the optimization function with respect to the results.

(3) The simulation results indicate that when the effect of the spiral case and substructure is ignored, the influence on the disturbance range continually increases as the correlation coefficient increases in the hydropower station model. The optimization function of the correlations between parameters is too important to be neglected when calculating the disturbance range.

(4) In conclusion, the analysis of the proposed model shows that the effect of the water turbine head cover is not evident in the vertical vibration transfer, but its transfer efficiency is significant.

The analysis of vibration transfer paths of hydropower station units and powerhouses is complicated. Based on the complex disturbances and parameters, the contribution of each transfer path can be calculated by analyzing the sensitivity of the transfer force and the transmissibility of the vibration model in the frequency domain. This study provides a reference for future comprehensive research on the transfer paths of hydropower station units and powerhouses.

\section{Acknowledgment}

This research was sponsored by the National Natural Science Foundation of China (51079020).

\section{References}

[1] Z. Y. Ma and Y. X. Dong, Dynamics of Water Turbine Generator Set, Dalian University of Technology Press, Dalian, China, 1st edition, 2003.

[2] Z. Y. Ma and Y. X. Dong, Vibration and Its Corrective Actions of Water Turbine Generator Set and Power House, China Water Power Press, Beijing, China, 1st edition, 2004.

[3] B. N. Singh, D. Yadav, and N. G. R. Iyengar, "Natural frequencies of composite plates with random material properties using higher-order shear deformation theory," International Journal of Mechanical Sciences, vol. 43, no. 10, pp. 2193-2214, 2001.

[4] T. D. Popescu, "Detection and diagnosis of model parameter and noise variance changes with application in seismic signal processing," Mechanical Systems and Signal Processing, vol. 25, no. 5, pp. 1598-1616, 2011.

[5] J. D. Kaplunov, E. V. Nolde, and B. F. Shorr, "A perturbation approach for evaluating natural frequencies of moderately thick elliptic plates," Journal of Sound and Vibration, vol. 281, no. 3-5, pp. 905-919, 2005.

[6] M. Kamiński, "Least squares stochastic boundary element method," Engineering Analysis with Boundary Elements, vol. 35, no. 5, pp. 776-784, 2011.

[7] M. Madani, M. Fathizadeh, Y. Khan, and A. Yildirim, "On the coupling of the homotopy perturbation method and Laplace transformation," Mathematical and Computer Modelling, vol. 53, no. 9-10, pp. 1937-1945, 2011.

[8] J. D. Collins and W. T. Thompson, "Eigenvalue problem for structural systems with statistical properties," AIAA Journal, vol. 7, no. 4, pp. 642-648, 1969.

[9] T. Hisada and S. Nakagiri, "Stochastic finite element analysis of uncertain structural systems," in Proceedings of the 4th International Conference in Australia on Finite Element Methods in Engineering, pp. 133-137, 1982.

[10] W. K. Liu, G. Bestefield, and T. Belytschko, "Vibrational approach to probabilistic finite elements," Journal of Engineering Mechanics-ASCE, vol. 114, no. 12, pp. 2115-2133, 1988.

[11] W. J. Vetter, "Matrix calculus operations and Taylor expansions," SIAM Review, vol. 15, pp. 352-369, 1973.

[12] H. Poincare, New Methods of Celestial Mechanics, Also History of Modern Physics and Astronomy, Washington, DC, USA, 1st edition, 1960. 
[13] E. A. Frieman, "On a new method in the theory of irreversible processes," Journal of Mathematical Physics, vol. 4, pp. 410-418, 1963.

[14] A. H. Nayfeh, "A perturbation method for treating nonlinear oscillation problems," vol. 44, pp. 368-374, 1965.

[15] N. Krylov and N. N. Bogoliubov, Intrduction to Nonlinear Mechanics, Princeton University Press, Princeton, NJ, USA, 1st edition, 1947.

[16] N. N. Bogoliubov and Y. A. Mitropolsky, Asymptotic Methods in the Theory of Nonlinear Oscillations, Gordon and Breach Science, New York, NY, USA, 1st edition, 1961.

[17] S. Kaplun, Fluid Mechanics and Singular Perturbations, P. A. Lagerstrom, L. N. Howard and Ching-shi Liu, Eds., Academic Press, New York, NY, USA, 1st edition, 1967.

[18] H. S. Tsien, “The Poincare-Lighthill-Kuo method," Advances in Applied Mechanics, vol. 4, pp. 281-293, 1956.

[19] S. Abbasbandy, "The application of homotopy analysis method to nonlinear equations arising in heat transfer," Physics Letters A, vol. 360, no. 1, pp. 109-113, 2006.

[20] Y. Zhang, S. Chen, Q. Liu, and T. Liu, "Stochastic perturbation finite elements," Computers and Structures, vol. 59, no. 3, pp. 425-429, 1996.

[21] Y. M. Zhang, "Frequency domain transfer degree of vibration transmission path ordering," Progress in Natural Science, vol. 17, no. 3, pp. 410-414, 2007.

[22] Y. M. Zhang, Q. Liu, and B. Wen, "Practical reliability-based design of gear pairs," Mechanism and Machine Theory, vol. 38, no. 12, pp. 1363-1370, 2003.

[23] Y. M. Zhang and Z. Yang, "Reliability-based sensitivity analysis of vehicle components with non-normal distribution parameters," International Journal of Automotive Technology, vol. 10, no. 2, pp. 181-194, 2009.

[24] W. Zhao and Y.-M. Zhang, "Response of vibration transfer path systems with uncertain paths," Journal of Vibration and Shock, vol. 28, no. 2, pp. 99-101, 2009.

[25] W. Gao, "Interval natural frequency and mode shape analysis for truss structures with interval parameters," Finite Elements in Analysis and Design, vol. 42, no. 6, pp. 471-477, 2006.

[26] W. Gao, "Natural frequency and mode shape analysis of structures with uncertainty," Mechanical Systems and Signal Processing, vol. 21, no. 1, pp. 24-39, 2007.

[27] W. Gao, J. J. Chen, T. B. Hu, N. J. Kessissoglou, and R. B. Randall, "Optimization of active vibration control for random intelligent truss structures under non-stationary random excitation," Structural Engineering and Mechanics, vol. 18, no. 2, pp. 137-150, 2004.

[28] J. Ma, J. J. Chen, W. Gao, and Y. Y. Zhao, "Stationary random response analysis of linear fuzzy truss," Structural Engineering and Mechanics, vol. 22, no. 4, pp. 469-481, 2006.

[29] J. Ma, J.-J. Chen, W. Gao, and T.-S. Zhai, "Non-stationary stochastic vibration analysis of fuzzy truss system," Mechanical Systems and Signal Processing, vol. 20, no. 8, pp. 1853-1866, 2006.

[30] J. Ma, W. Gao, P. Wriggers, T. Wu, and S. Sahraee, "The analyses of dynamic response and reliability of fuzzy-random truss under stationary stochastic excitation," Computational Mechanics, vol. 45, no. 5, pp. 443-455, 2010.

[31] H. Shinzawa, T. Genkawa, and W. Kanematsu, "Pressureinduced association of oleic acid (OA) under varying temperature studied by multiple-perturbation two-dimensional (2D) IR correlation spectroscopy," Journal of Molecular Structure, vol. 1028, pp. 164-169, 2012.
[32] B. Allés, A. Buonanno, and G. Cella, "Perturbation theory predictions and Monte Carlo simulations for the $2 \mathrm{D} \mathrm{O}(\mathrm{n})$ nonlinear $\sigma$-models," Nuclear Physics B, vol. 500, no. 1-3, pp. 513$543,1997$.

[33] C. J. Butte, Y. Zhang, H. Song, and J. J. Jiang, "Perturbation and nonlinear dynamic analysis of different singing styles," Journal of Voice, vol. 23, no. 6, pp. 647-652, 2009.

[34] L. Chai, A. J. Sprecher, Y. Zhang, Y. Liang, H. Chen, and J. J. Jiang, "Perturbation and nonlinear dynamic analysis of adult male smokers," Journal of Voice, vol. 25, no. 3, pp. 342-347, 2011.

[35] A. Pirrotta, "Non-linear systems under delta correlated processes handled by perturbation theory," Probabilistic Engineering Mechanics, vol. 13, no. 4, pp. 283-290, 1998.

[36] E. G. D'Ambrogio, “The dynamics of complex stochastic models evolution of a correlation function with random fluctuations," Mathematical and Computer Modelling, vol. 28, no. 3, pp. 15-20, 1998.

[37] N. A. Husain, H. H. Khodaparast, and H. Ouyang, "Parameter selection and stochastic model updating using perturbation methods with parameter weighting matrix assignment," Mechanical Systems and Signal Processing, vol. 32, pp. 135-152, 2012.

[38] H. H. Khodaparast, J. E. Mottershead, and M. I. Friswell, "Perturbation methods for the estimation of parameter variability in stochastic model updating," Mechanical Systems and Signal Processing, vol. 22, no. 8, pp. 1751-1773, 2008.

[39] W. Gao, C. Song, and F. Tin-Loi, "Probabilistic interval response and reliability analysis of structures with a mixture of random and interval properties," Computer Modeling in Engineering of Sciences, vol. 46, no. 2, pp. 151-189, 2009.

[40] B. Arazi, "Some properties of Hadamard matrices generated recursively by Kronecker products," Linear Algebra and its Applications, vol. 25, pp. 27-39, 1979.

[41] D. H. Zhao, Y. X. Dong, and Z. Y. Ma, "Static and dynamic analysis for concrete supporting structure of turbine unit," Hydroelectric Energy Science, vol. 18, no. 4, pp. 68-71, 2000.

[42] R. Cardinali, R. Nordmann, and A. Sperber, "Dynamic simulation of non-linear models of hydroelectric machinery," Mechanical Systems and Signal Processing, vol. 7, no. 1, pp. 29-44, 1993. 


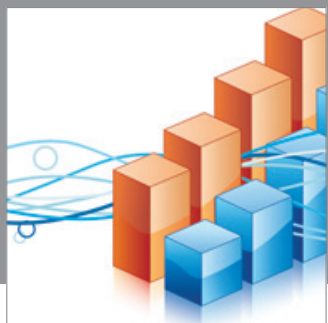

Advances in

Operations Research

mansans

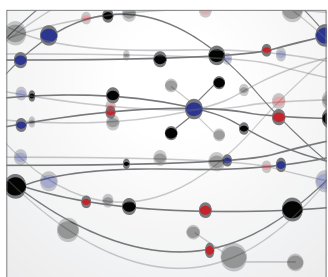

The Scientific World Journal
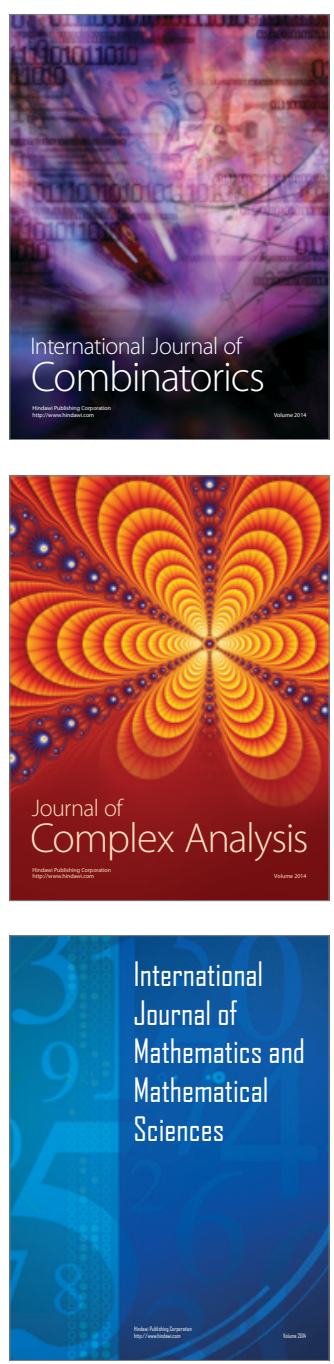
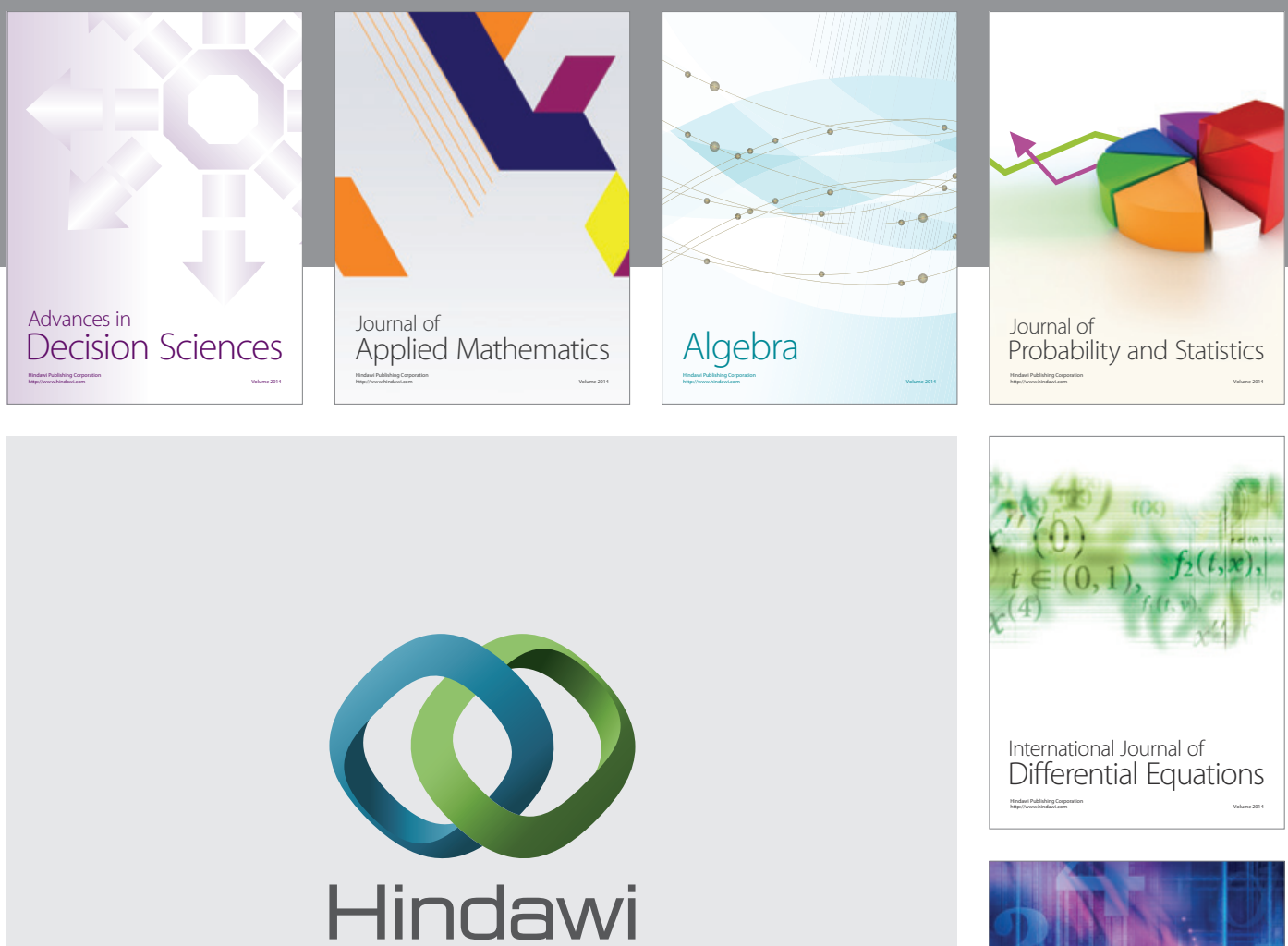

Submit your manuscripts at http://www.hindawi.com
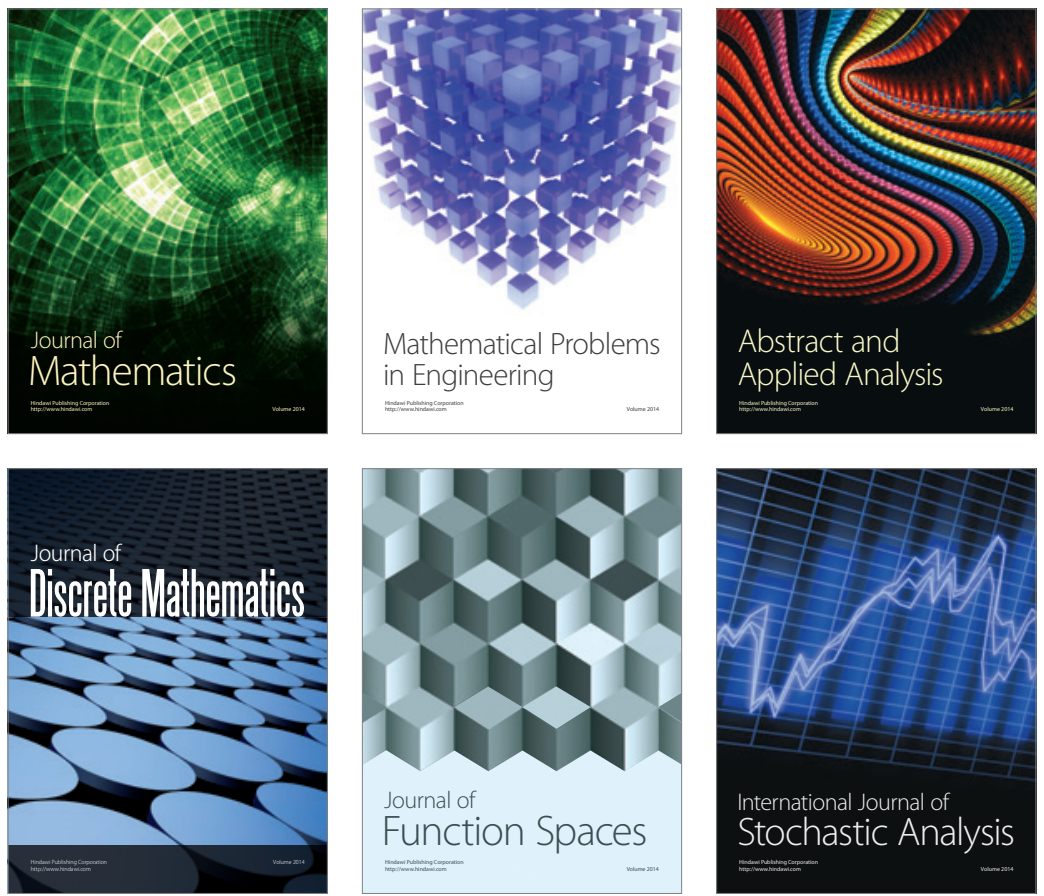

Journal of

Function Spaces

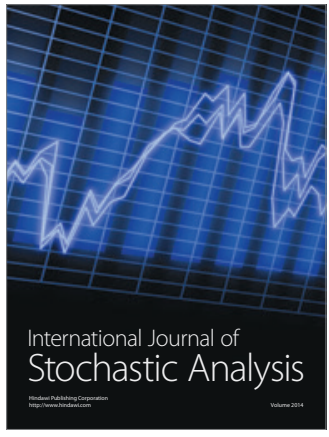

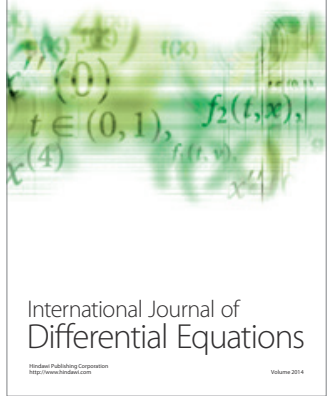
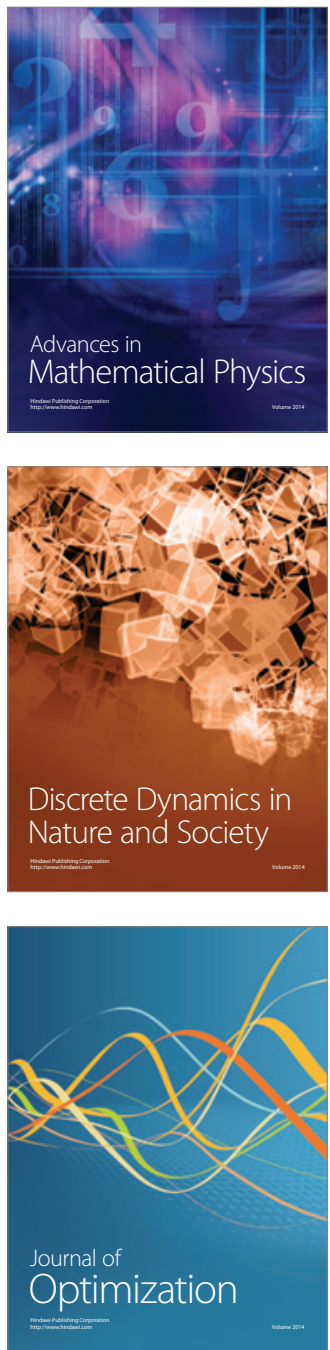